\title{
Mucoepidermoid carcinoma of the bronchus: a rare early diagnosis
}

\author{
Rohit Kumar ${ }^{1}$, Harsh Vardhan Puri ${ }^{2}$, Nitesh Gupta ${ }^{1}$, Sukhram Bishnoi ${ }^{2}$, Mohan Venkatesh Pulle ${ }^{2}$, \\ Pranav Ish ${ }^{1}$ \\ ${ }^{1}$ Department of Pulmonary, Critical Care and Sleep Medicine, Vardhman Mahavir Medical College and Safdarjung \\ Hospital, New Delhi; ${ }^{2}$ Department of Thoracic Surgery, Sir Ganga Ram Hospital, New Delhi, India
}

\begin{abstract}
Salivary gland tumours of the tracheobronchial tree are rare and early diagnosis in $\mathrm{T} 1$ stage is further rare. We report a case of a young 21-year-old male medical student diagnosed and treated for the same prompted by a detailed respiratory examination.
\end{abstract}

\section{Case Report}

The stethoscope is becoming more of a ceremony than utility. However, each clinical tool has its own utilities and the importance of auscultation cannot be over-emphasized.

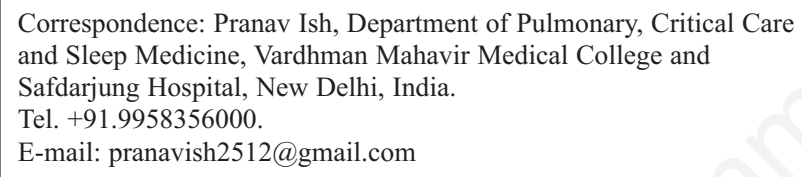

Contributions: All the authors contributed to substantial contributions to the conception or design of the work; the acquisition, analysis, or interpretation of data for the work; drafting the work or revising it critically for important intellectual content; final approval of the version to be published. All the authors agree to be accountable for all aspects of the work in ensuring that questions related to the accuracy or integrity of any part of the work are appropriately investigated and resolved.

Consent for publication: The patient gave his written consent to use his personal data for the publication of this case report and any accompanying images. The patient understands that his name and initials will not be published, but that anonymity cannot be guaranteed.

Conflict of interest: The authors declare that they have no competing interests, and all authors confirm accuracy.

Key words: mucoepidermoid carcinoma; trachea; rare; stethoscope.

Received for publication: 26 February 2020.

Accepted for publication: 19 March 2020.

${ }^{\circ}$ Copyright: the Author(s), 2020

Licensee PAGEPress, Italy

Monaldi Archives for Chest Disease 2020; 90:1258

doi: 10.4081/monaldi.2020.1258

This article is distributed under the terms of the Creative Commons Attribution Noncommercial License (by-nc 4.0) which permits any noncommercial use, distribution, and reproduction in any medium, provided the original author(s) and source are credited.
A medical intern presented to the pulmonary outpatient department (OPD) with complaints of episodic wheeze 1-day prior with no other symptoms. There was no fever, cough, sputum, haemoptysis, or dyspnoea. On examination, he had preserved vitals but on auscultation, breath sounds were slightly reduced on the left interscapular and intrascapular area with localised wheeze. A chest radiograph was performed (Figure 1A) and reported normal. A short acting bronchodilator was prescribed and patient was asymptomatic. However, the slight decrease in breath sound intensity confirmed with decreased vocal resonance prompted the pulmonologist to advice a CT chest (Figure $1 \mathrm{~B}, \mathrm{C}$ ) which revealed an intraluminal growth of size $1 \times 1 \mathrm{~cm}$ in left main bronchus. A differential diagnosis of inflammatory myofibroblastic tumour, polyp or carcinoid were kept. Fibreoptic bronchoscopy revealed a sessile growth in left main bronchus (Figure 2A) with normal distal segmental anatomy. Endobronchial biopsy was suggested of mucoepidermoid carcinoma (MEC) (Figure 2B) confirmed by immunohistochemistry (Figure 2C).

A multidisciplinary discussion regarding the treatment options were discussed. The patient underwent a sleeve resection (Figure 3A) of left main bronchus with intraoperative frozen section of margins and systematic mediastinal lymph node dissection. Histopathology showed a T1a mucoepidermoid carcinoma of $1 \mathrm{~cm}$ size with negative margins and sixteen regional lymph nodes free of tumour. Post-operative chest X-ray (Figure 3B) revealed completely expanded lung with intercostal drain in situ. The patient was discharged on day 4 of hospitalisation and post intercostal drain removal. Follow up chest X-ray on day 7 (Figure 3C) showed complete lung expansion. The patient had equal breath sounds in bilateral lung fields on discharge.

\section{Discussion}

Primary salivary-gland comprise only $0.1-0.2 \%$ of primary lung cancers and not even $1 \%$ of primary malignant bronchial tumours [1]. MEC originates from the submucosal glands of the tracheobronchial tree. Pulmonary MECs are commonly found in the segmental or lobar bronchi, rarely presenting as endobronchial lesions. It affects patients of age 30 years and older [2]. Our patient was a rare early diagnosis with pathological stage T1a resected successfully. Patients may present with cough, haemoptysis, wheeze [3] and post-obstructive pneumonia, but may also be asymptomatic [4]. These symptoms are frequently misdiagnosed as asthma, bronchitis or pneumonia [5]. This is a rare case of low grade mucoepidermoid carcinomas of the bronchus.

Surgical resection is the mainstay of treatment, and usually carries good prognosis. Low-grade malignant tumours of the bronchial airway are often managed with surgical resection. High- 
grade MEC carries a higher risk of distant metastasis and worse prognosis even with surgery, hence, adjuvant chemotherapy and radiotherapy may be beneficial [6].

A recent review of over 8 years including salivary gland tumour of trachea-bronchial tree had only 16 cases of MEC. Most patients treated with surgery, it was found more cases of MEC

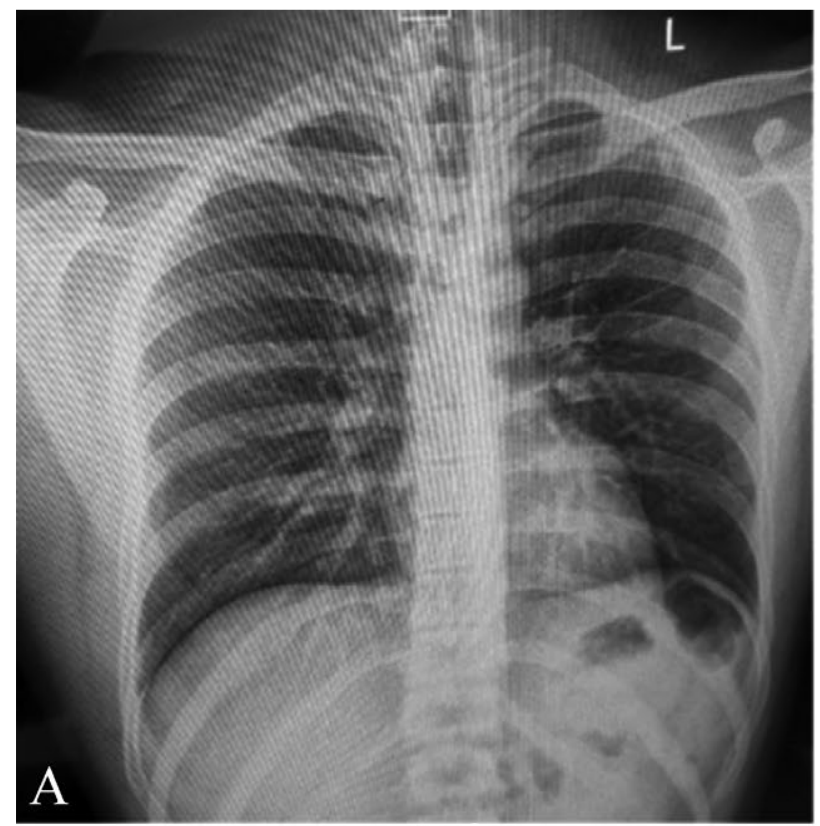

associated with negative surgical margins as compared to adenoid cystic carcinoma (ACC), though there was no difference in terms of recurrence. The review concluded that currently, the images and clinical features remain unclear because of the rarity of these tumours. It revealed that patients with MEC commonly had a good prognosis, but the disease easily leads to a misdiagnosis due to its rarity and few characteristic clinical features [7]

A large cytopathological review of salivary gland tumour of tracheobronchial tree revealed only 11 patients, with MEC being the $2^{\text {nd }}$ most common after AEC diagnosed by biopsy or needle aspiration. All patients were treated with surgery and confirmed the diagnosis [5].

A large series from India reported 7 cases, most of which underwent radical surgeries involving pneumonectomy and bilobectomy. However, these patients had large tumours with haemoptysis and dyspnoea [8]. Lymphatic involvement even in low grade tumours necessitates non-conservative surgeries [9]. Fortunately, our patient had an early diagnosis and had no lymphatic involvement radiologically confirmed pathologically.

The stethoscope is one of the most powerful tools of the physician. Even in a large study comparing stethoscope with lung ultrasound, it was found that ultrasound has poor correlation and fails to detect pulmonary edema in patients having crepitations and rhonchi. A subgroup analysis showed that diagnostic accuracy of stethoscope auscultation was better specially in non-ventilated patients [10] Thus clinical history and detailed examination can not be replaced by modern facilities, rather should be assisted by them. The science and practice of auscultation should not be forgotten.
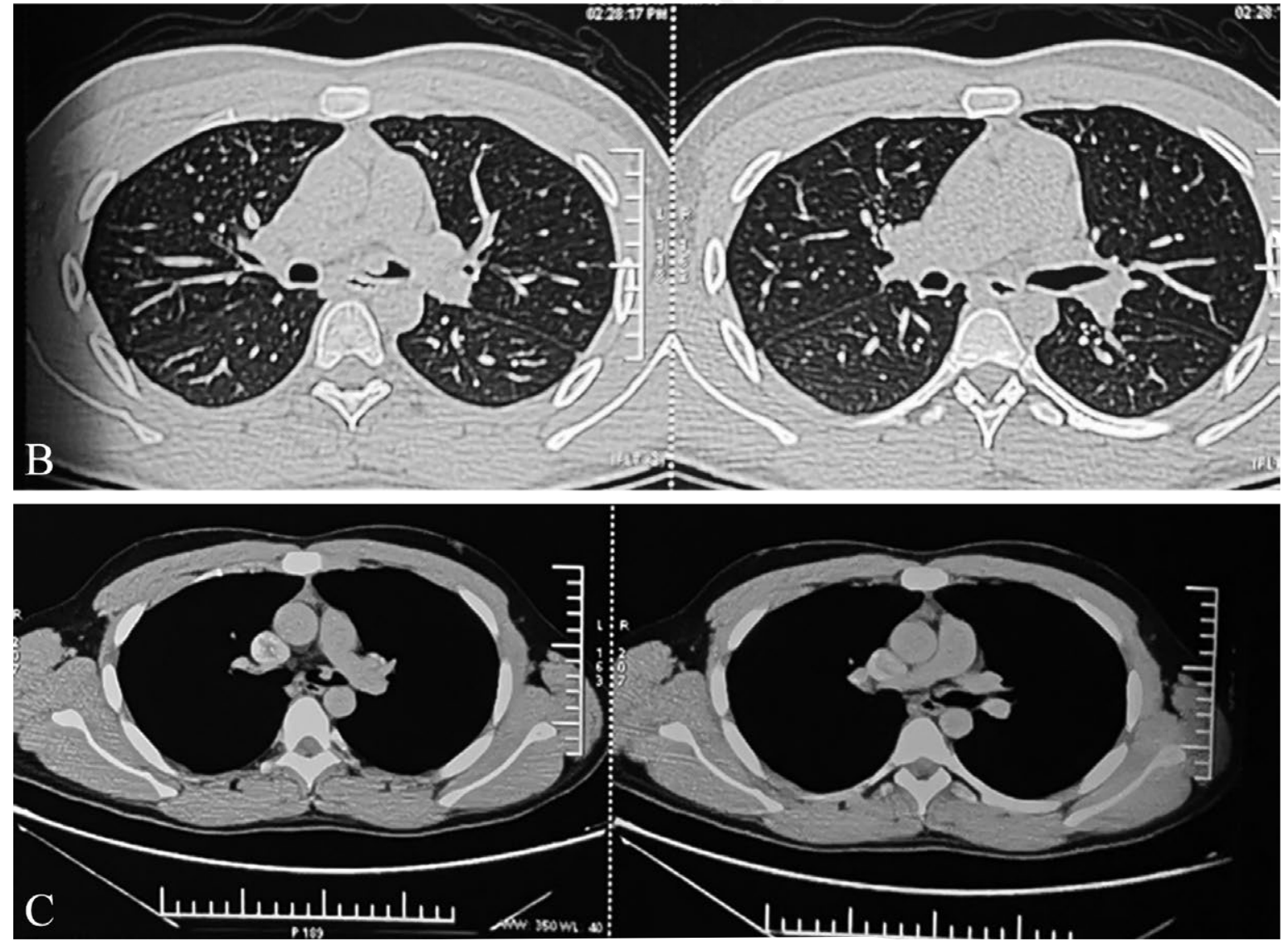

Figure 1. A) Chest X-ray posterior-anterior view revealing no abnormality. B,C) Contrast enhanced CT chest showing a well-defined rounded lesion arising from anterior wall of left main bronchus with no internal calcification or fat. 
To conclude, endobronchial MEC is a rare type of salivary gland tumour. Patients with low-grade MECs have a good prognosis. Early identification and surgical resection can result in a good prognosis. The importance of clinical examination by a stethoscope to look for subtle signs cannot be over-emphasized. A misdiagnosis or a delayed diagnosis of such tumours can lead to increased morbidity and mortality.
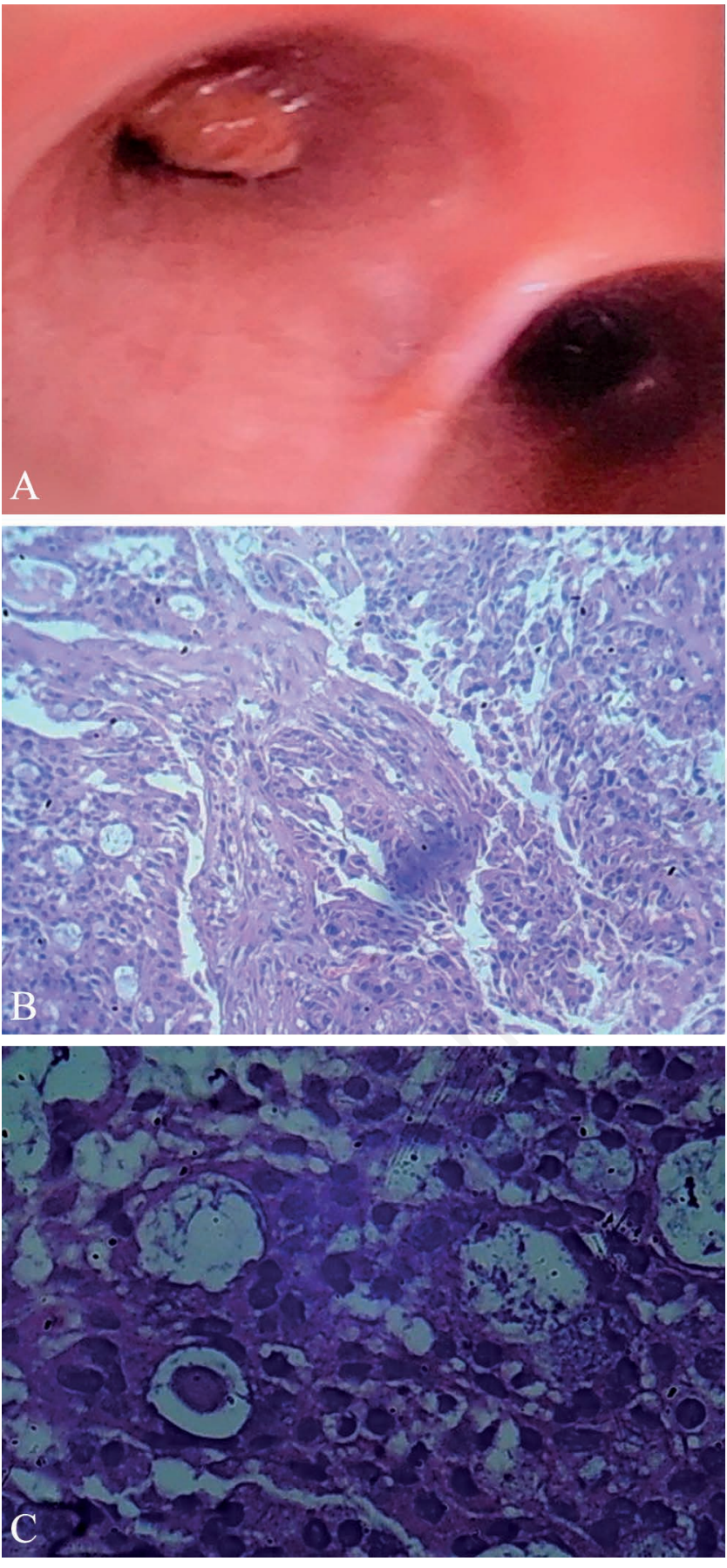

Figure 2. A) Fibreoptic bronchoscopy revealing a sessile intrabronchial growth in left main bronchus distal to carina. B,C) $40 x$ and 100x magnification with $H \& E$ staining of endobronchial biopsy showing pseudostratified ciliated columnar epithelium with atypical squamoid cells arranged in nests with blue cytoplasm and nuclear atypia.
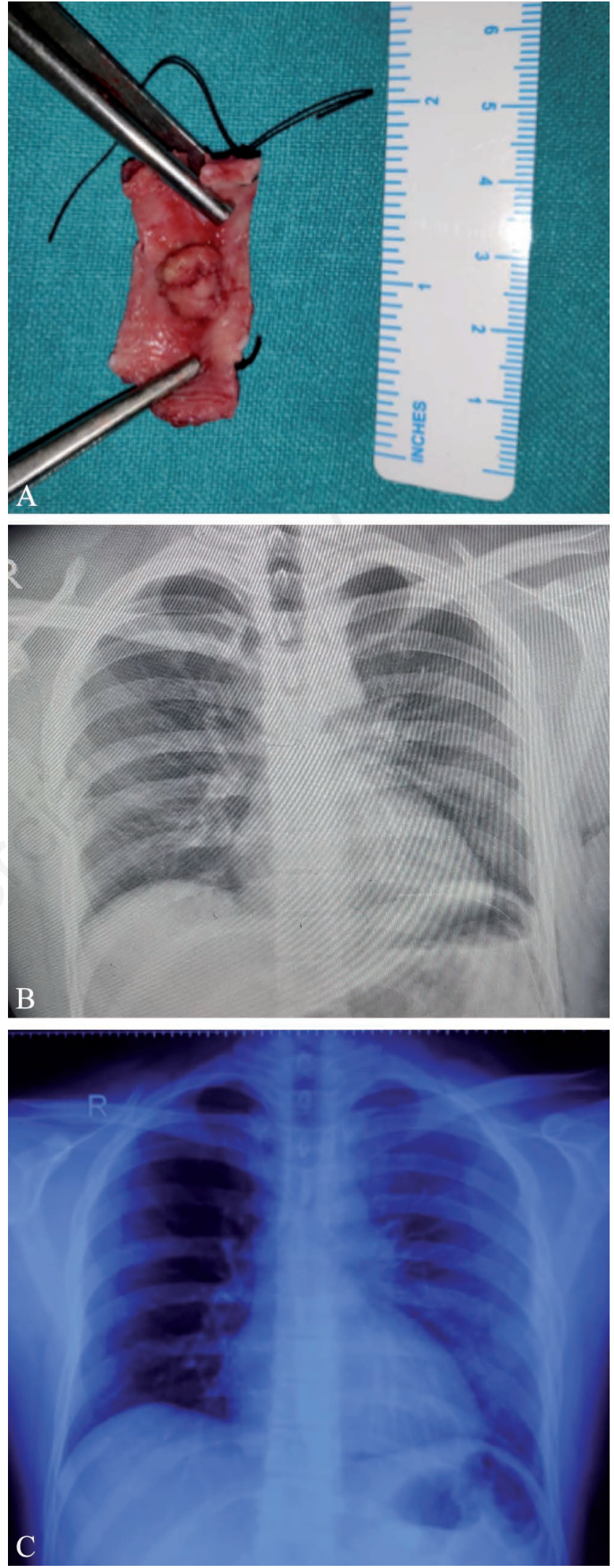

Figure 3. A) Post operative specimen showing $1 \times 1 \mathrm{~cm}$ lesion along with healthy margin of left bronchus removed by sleeve resection. B) Immediate post-operative chest X-ray with left intercostal drainage tube in situ with minimal pneumothorax. C) Post operative day 7 chest $\mathrm{X}$-ray showing normal lung fields. 


\section{References}

1. Abu Saleh WK, Aljabbari O, Ramchandani M. Mucoepidermoid carcinoma of the tracheobronchial tree. Methodist Debakey Cardiovasc J 2015;11:192-4.

2. Shen C, Che G. Clinicopathological analysis of pulmonary mucoepidermoid carcinoma. World J Surg Oncol 2014;12:33.

3. Lin $\mathrm{CH}$, Chao $\mathrm{YH}, \mathrm{Wu} \mathrm{KH}$, Lin WC. Primary mucoepidermoid carcinoma at the carina of trachea presenting with wheezing in an asthmatic child mimicking an attack of asthma: A case report. Medicine (Baltimore) 2016;95:e5292.

4. Al-Halawani M, Abdeen Y. Mucoepidermoid carcinoma of the trachea. Quant Imaging Med Surg. 2018;8:259-60.

5. Omesh T, Gupta R, Saqi A, et al. A rare case of endobronchial mucoepidermoid carcinoma of the lung presenting as nonresolving pneumonia. Respir Med Case Rep 2018;25:154-7.
6. Moon EJ, Kim BY, Chung JY, et al. Rare tracheal mucoepidermoid carcinoma with invasion of thyroid gland initially misdiagnosed as invasive thyroid cancer. Int $\mathrm{J}$ Clin Exp Med 2016;9:8874-82.

7. Han X, Zhang J, Fan J, et al. Radiological and clinical features and outcomes of patients with primary pulmonary salivary gland-type tumors. Can Respir J 2019;2019:1475024.

8. Pandey D, Garg PK, Jakhetiya A, et al. Surgical experience of primary salivary gland tumors of lung: A case series. Int J Surg 2015;21:92-6.

9. Menon P, Singh M, Rao KL, et al. Mucoepidermoid carcinoma of the bronchus: is conservative surgery always justified? Indian J Cancer 2003;40:34-6.

10. Cox EGM, Koster G, Baron A, et al. Should the ultrasound probe replace your stethoscope? A SICS-I sub-study comparing lung ultrasound and pulmonary auscultation in the critically ill. Crit Care 2020;24:14. 